\title{
PIN: Measuring Asymmetric Information in Financial Markets with $\mathbf{R}$
}

by Paolo Zagaglia

\begin{abstract}
The package PIN computes a measure of asymmetric information in financial markets, the so-called probability of informed trading. This is obtained from a sequential trade model and is used to study the determinants of an asset price. Since the probability of informed trading depends on the number of buy- and sell-initiated trades during a trading day, this paper discusses the entire modelling cycle, from data handling to the computation of the probability of informed trading and the estimation of parameters for the underlying theoretical model.
\end{abstract}

\section{Introduction}

A large set of finance packages are available in $\mathrm{R}$ for an array of tasks including, among others, portfolio analysis (e.g., see Boudt et al., 2010; Kane et al., 2011). Only a small set of tools is available for the study of intra-daily transactions, mostly focused on data-management issues. Yet these represent only the first building block for modelling the dynamics of asset prices.

In recent years, the rise of algorithmic and high-frequency trading has shed a bright light on the fact that movements in financial market prices are largely affected by microstructure forces. In particular, some traders in the market may enjoy privileged information about the value of an asset, while others may be trading merely on the basis of public news. The risk that an individual uninformed trader may face an informed counterparty in the market-place is then an important determinant of asset prices (e.g., see Easley et al., 2002). Hence, measuring the probability that a counterparty enjoys asymmetric information, also known as probability of informed trading (PIN), allows a trader to correctly price a security (see Easley and O'Hara, 1987).

The PIN package (Zagaglia, 2013) provides tools for calculating the probability of informed trading proposed by Easley et al. (1996). This measure is obtained by estimating a model of strategic interaction between traders with different information sets. In particular, the probability of informed trading depends on the number of buy- and sell-trades taking place in the market.

Knowledge about whether a trade is initiated by the buying or selling party is not always available. In this case, the so-called signing of trades can be obtained from applying alternative classification algorithms on trade and quote data, when available (e.g., see Lee and Ready, 1991). These functionalities are already offered by excellent packages in $\mathrm{R}$.

To illustrate the complementarity between these data-handling tools and the PIN package, I present a sample application that uses the highfrequency package of Cornelissen et al. (2013). I consider a data set of fictitious trades and quotes for a given trading day that is well representative of standard limited order books. This allows me to consider a preliminary issue surrounding the availability of data. Quotes often need to be matched to the available trades, in the sense of making the time stamps of the two consistent with each other. Only after this is done, can the user compute the number of implicit buy- and sell-trades, estimate the parameters of the sequential trade model of Easley et al. (1996), and compute the resulting PIN. In other words, I discuss the entire empirical modelling cycle for the probability of informed trading.

I should stress that typical applications of the PIN focus on the stock market. This is mostly due to the large availability of accessible data. However, recent empirical contributions have estimated the PIN for alternative markets, such as the U.S. government bond market (see Li et al., 2009). Idier and Nardelli (2011) provide an application to the European overnight money market. This is an over-thecounter market, for which only representative price quotes are available. Estimating the probability of informed trading on markets that do not feature a transparent organization of exchanges-for instance, in the form of an order book or a market-maker-requires adapting the model of Easley et al. (1996) to take into account the relevant idiosyncratic features. For this reason, the discussion in this paper focuses on standard market structures, such as those of today's stock markets, and disregards the more controversial case of over-the-counter markets.

\section{$\mathbf{R}$ packages for market microstructure analysis}

The basic prerequisite for any application in market microstructure is the availability of proper financial data. Hence, the R developers' community has proposed a number of efficient and powerful tools to address the resulting big-data challenge. 


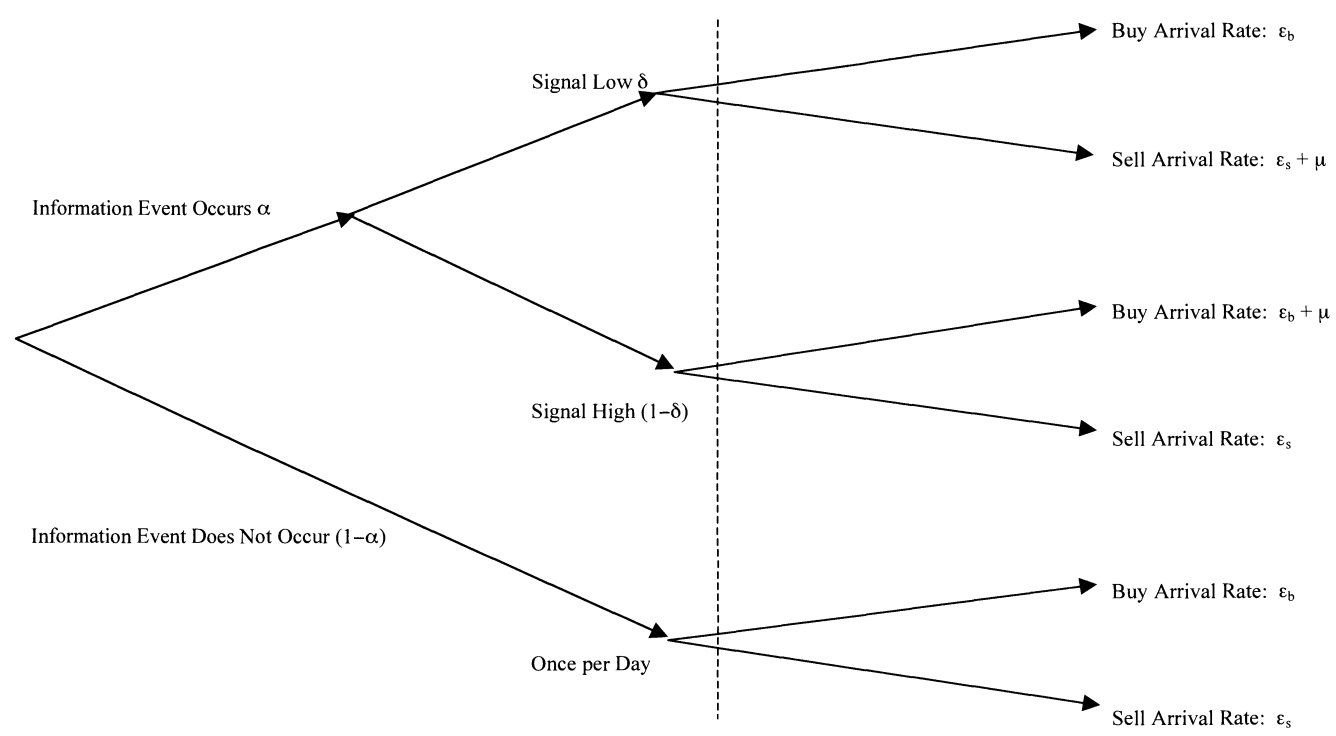

Figure 1: The trading game

The starting point consists in downloading the data set from a provider. The package IBrokers by Ryan (2011) provides access to the data-feed supplied by Interactive Brokers and automates data requests for the creation of high-frequency data sets.

Given the availability of a financial-market data set, a variety of excellent functions are available in $\mathrm{R}$ for data management, including data re-shuffling, plotting and computing descriptive statistics. Kane et al. (2011) presents the package orderbook, which provides data-handling functionalities from limited order books. Boudt and Cornelissen (2012) present $\mathrm{R}$ functions for managing the trade and quote (TAQ) data set from NASDAQ and NYSE in R. TAQ data set files are available in different forms that report different types of information, potentially involving a detailed structure of the order books. This can easily result in terabytes of data. For this purpose, Emerson and Kane have extended the available packages with the aim of handling terabyte-large data sets ${ }^{1}$.

Only a few tools are available to the user community for the study of market-microstructure issues in R. Enos et al. (2008) present a library for the computation of trade costs in market transaction of stocks. In a recent conference presentation ${ }^{2}$, Yan and Zivot discuss the implementation of models for price impact and discovery in the stock market.

The RTAQ package has recently been incorporated into the highfrequency package (Cornelissen et al., 2013). In this extension, the user is provided with functions to compute different measures of market liquidity, such as those of Hasbrouck and Seppi (2001) and Venkataraman (2001). This package also provides functionalities for classifying trade origination. Not all the markets provide public information on whether a trade starts either from the buy-side, or from the sell-side. This is a prerequisite for studying a large number of topics in market microstructure, including asymmetric information and informed trading. For this purpose, several methods have been proposed to infer trade direction from changes in market prices and quotes. The highfrequency package implements an effective modification of the so-called tick rule proposed by Lee and Ready (1991).

\section{Computing the probability of informed trading}

\section{The theoretical model}

Easley et al. (1996) build on the sequential trading model of Glosten and Milgrom (1987) and Kyle (1985) to estimate the probability that a counterpart in the trading process enjoys superior information. This is based on the assumption that the market is composed by a heterogeneous population of traders. Some traders are informed, while others are uninformed.

Within each trading day, a competitive market-maker buys or sells a security at the bid and ask

\footnotetext{
${ }^{1}$ Towards Terabytes of TAQ, R in Finance 2012, http: / / www. rinfinance. com/agenda/2012/talk/Emerson+Kane. pdf

${ }^{2}$ Estimating the Dynamics of Price Discovery, R in Finance 2012, http: //www . rinfinance. com/agenda/2012/ talk/Yan+Zivot.pptx
} 
quotes that he or she announce publicly to the market. Since the market-maker is risk-neutral and competes with the traders in the transactions, the resulting prices represent the expected value of the stock conditional on the market-maker's information at the time of trade.

Before the beginning of a trading day, nature determines whether an information event relevant to the value of the stock will occur. Information events are independently distributed and occur with probability $\alpha$. Good news events take place with a probability $\delta$ and bad news events occur with a probability $1-\delta$. At the end of the day, the information set of each actor in the market is complete and the true value of the stock is realized.

The market-maker knows both the probability of these events taking place and the arrival order of information to the market. However, he or she does not know which event is realized. Easley et al. (1996) assume that the market-maker is Bayesian in that they use the information from trade arrival to update their belief about information events. Since the information set is independent across days, the model structure allows the change in beliefs to be considered separately on each day.

In this framework, the market is made by the traders that can observe the signals about the true stock value and that benefit from asymmetric information. There are also uninformed traders that receive no news about the stock price. These two groups of traders enter the market with a frequency determined by independent Poisson processes at any minute within a trading day.

An information-revealing event causes informed traders to enter the market. For instance, if a 'high-value' or 'good' signal materializes, these traders buy the stock. The arrival of news events both to the market and to each trader follow independent Poisson processes.

The set of events and associated probabilities can be represented in the trading tree of Figure ??. At the first node of the tree, nature selects whether an information event occurs. If an event does occur, nature determines the sign of the resulting news. The three nodes of 'no event', 'good news' and 'bad news' placed before the dotted line of Figure ?? occur only once per day. Given the node selected for the day, traders arrive according to the relevant Poisson processes. On good-event days, the arrival rates are $\epsilon+\mu$ for buy orders and $\epsilon$ for sell orders. On bad-event days, the arrival rates are $\epsilon$ for buys and $\epsilon+\mu$ for sells. Finally, on days without information-revealing news, only uninformed traders take part in the market with an arrival rate of $\epsilon$.

While observing changes in the order book, traders obtain information about order arrival. However, they cannot observe the underlying motives for trading by counterparties. In other words, traders do not know the parameter values of the model consistent with trading patterns. However, these parameters can be estimated from data on order arrivals.

\section{Empirical implementation of the theoretical model}

In order to reduce the parameter space, it is typically assumed that the sell- and buy-arrival rates $\epsilon_{b}$ and $\epsilon_{s}$ are equal. On a good-day event, the probability of observing a sequence of buy- and sell-initiated trades is given by

$$
e^{-(\mu+\epsilon) T} \frac{[(\mu+\epsilon) T]^{B}}{B !} e^{\epsilon T} \frac{(\epsilon T)^{S}}{S !}
$$

On a bad-signal day, this probability becomes

$$
e^{\epsilon T} \frac{(\epsilon T)^{B}}{B !} e^{-(\mu+\epsilon) T} \frac{[(\mu+\epsilon) T]^{S}}{S !}
$$

Finally, when information-revealing events do not take place, the likelihood of observing orders is equal to

$$
e^{\epsilon T} \frac{(\epsilon T)^{B}}{B !} e^{\epsilon T} \frac{(\epsilon T)^{S}}{S !}
$$

Assuming that trading activity is independent across $T$ days, the likelihood of trading activity takes the form

$$
\begin{aligned}
\mathrm{L}[\{B, S\} \mid \theta]=(1-\alpha) e^{-\epsilon T} \frac{(\epsilon T)^{B}}{B !} e^{-\epsilon T} \frac{(\epsilon T)^{S}}{S !} & \\
& +\alpha \delta e^{-\epsilon T} \frac{(\epsilon T)^{B}}{B !} e^{-(\mu+\epsilon) T} \frac{[(\mu+\epsilon) T]^{S}}{S !} \\
& +\alpha(1-\delta) e^{-(\mu+\epsilon) T} \frac{[(\mu+\epsilon) T]^{B}}{B !} e^{-\epsilon T} \frac{(\epsilon T)^{S}}{S !}
\end{aligned}
$$

with the parameter space $\theta=\alpha, \delta, \epsilon, \mu$. Over $h$ independent days, the likelihood of observing the data 
$M=\left(B_{i}, S_{i}\right)_{i=1}^{h}$ is the product of the daily likelihoods

$$
\mathrm{L}[M \mid \theta]=\prod_{i=1}^{h} \mathrm{~L}\left(\theta \mid B_{i}, S_{i}\right)
$$

To deal with the issue of convergence in numerical maximization, I follow Aktasa et al. (2007) and Easley et al. (2008), and re-arrange the likelihood function 5 as:

$$
\begin{aligned}
\mathrm{L}[M \mid \theta]=\sum_{t=1}^{T}\left[-2 \epsilon+M_{t} \ln x\right. & \left.+\left(B_{t}+S_{t}\right) \ln (\mu+\epsilon)\right] \\
& +\sum_{t=1}^{T} \ln \left[\alpha(1-\alpha) e^{-m u} x^{S_{t}-M_{t}}+\alpha \delta e^{-\mu} x^{B_{t}-M_{t}}+(1-\alpha) x^{B_{t}+S_{t}-M_{t}}\right]
\end{aligned}
$$

with $M_{t}=\min \left(B_{t}, S_{t}\right)+\max \left(B_{t}, S_{t}\right) / 2$, and $x_{t}=\epsilon /(\mu+\epsilon)$.

Consistent with the literature (e.g., see Easley et al., 2002, 1996), the role of asymmetric information is measured by the probability of informed trading $\left(\mathrm{PIN}_{t}\right)$. This is defined as the unconditional probability that informed traders buy or sell an asset at each point in time, and is equal to

$$
\mathrm{PIN}_{t}=\frac{\alpha \mu}{\alpha \mu+2 \epsilon}
$$

When this probability is high, uninformed traders face a higher risk of trading with a counterparty that is better informed.

The package PIN includes an $\mathrm{R}$ function for the computation of the log-likelihood function 6. This takes as inputs the parameter values-in the order $\epsilon, \mu, \alpha, \delta$-and the time series of daily data on number of buy- and sell-initiated trades-arranged as a $n \times w$ matrix, where $n$ is the number of trading days. The first column of this data matrix includes the number of buy trades and the second one has the number of sell trades.

\section{A practical example}

In this section I present an example of how to integrate the PIN package with other tools available in $\mathrm{R}$ for handling market-microstructure data sets. In particular, the application presented here relies on the capabilities provided by the highfrequency package.

I discuss the computation of PIN using a data set of fictitious quotes and trades for an ideal stock. These quotes and trades are provided as two data sets-sample_qdata and sample_tdata, respectivelyin the public distribution of the package highfrequency. ${ }^{3}$

The noteworthy characteristic of the database for trades and quotes is that its structure follows the TAQ format for standard NYSE stocks. In other words, the approach discussed here is general enough to handle a data source that has been a key reference in the industry.

It should be stressed that the fictitious trades and quotes refer to one trading day only. This is an evident simplification, which is typically avoided in empirical studies of PIN. For instance, Easley et al. (2002) exclude stocks that have less than 60 days of quotes and trades data, suggesting that would disrupt the reliability of the model parameter estimates. The PIN package allows estimations over any sample length to be run. Moreover, the application presented in this paper can easily be extended to cover more than one trading day.

As a starting point, I load the relevant packages into R, including those required by the highfrequency package:

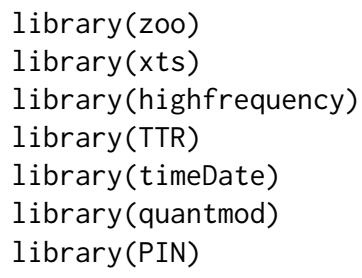

Then, I prepare the data set for the estimation of PIN. The highfrequency package includes two sample files with the trades and quotes. These two pieces of information need to be matched, in the sense of ordering the quoting activity that is consistent with the recorded trades within a given trading day:

${ }^{3}$ Hence, data for these fictitious trades and quotes are available online to the R community. 
\#Load data samples

data (sample_tdata)

data(sample_qdata)

\#Match the trade and quote data

tqdata <- matchTradesQuotes(sample_tdata, sample_qdata)

The data set used in this example does not include information on trade direction. As stressed earlier, this is an issue that is present in certain markets and can be addressed using the trade classification algorithms proposed in the finance literature. Here I use the functionality of the highfrequency package to apply the Lee-Ready approach that combines trading and quoting activity:

$x<-$ getTradeDirection(tqdata)

tradeDirection $<-\operatorname{matrix}(\mathrm{x})$

buy_side $<-$ which(tradeDirection $>0$ )

num_buy_side <- length(matrix(buy_side))

num_sell_side <- length(tradeDirection) - length(matrix(buy_side))

ntrades <- cbind(num_buy_side, num_sell_side)

The maximization of the log-likelihood function 6 is a prerequisite for the computation of the PIN. Ideally, one should perform a constrained optimization and restrict the spaces of all the parameters over the unit interval $[0,1]$. For this particular data set, I employ the general optimization routine optim:

initparams <- $\operatorname{cbind}(0.15,0.05,0.5,0.5)$

options (warn $=-1)$

param_optim <- optim(initparams, pin_likelihood, gr = NULL, ntrades)

The optimizing parameters are $1,0.3715623,0.7255069$ and 0.2808594 . These estimated values suggest that there is a high probability for the trading day to reveal information (i.e., $\hat{\alpha} \approx 0.72$. When there are no news events, only uninformed traders take part in the market $(\epsilon=1)$.

Finally, given the optimizing parameters, the computation of the probability of informed trading is straightforward:

epsi <- param_optim\$par[1]

miu <- param_optim\$par[2]

alph <- param_optim\$par[3]

delt <- param_optim\$par[4]

pin $<-($ alph*miu $) /($ alph*miu $+2 * e p s i)$

In this case, I obtain a low probability of trading with an informed counterparty, which is equal to 0.1187762

\section{Conclusion}

This article discusses an application in $\mathrm{R}$ of concepts and methods of financial market microstructure. In particular, I present a package for the computation of the probability of informed trading. This is a standard measure of asymmetric information that is based on trade direction. I show how the proposed PIN package fits into the broader context of the available tools for handling microstructure data.

The routines outlined here could be extended along several dimensions. It would be useful to include alternative measures of information asymmetry. The time-varying PIN specification of Easley et al. (2008) represents a relevant candidate. Also, the Volume-Synchronized Probability of Informed Trading - VPIN - is a key information metric that is increasingly becoming the industry standard (see Easley et al., 2012).

The provision of liquidity to the market is related to the presence of asymmetric information. This consideration is especially relevant for market-makers, who manage inventories against the risk that counterparties have superior information on the true value of an asset. Hence, any tool that computes the PIN provides an incomplete picture of the market if it says nothing about the sources for 
market liquidity. For this reason, it would be beneficial to provide tools for the measurement of price pressures, such as those proposed by Hendershott and Menkveld (1991) for the NYSE.

\section{Acknowledgements}

The author is very grateful to two anonymous referees for very effective comments that have shaped the revision of this article to a major extent.

\section{Bibliography}

N. Aktasa, E. de Bodta, F. Declerckc, and H. Van Oppensa. The PIN Anomaly around M\&A Announcements. Journal of Financial Markets, 10(2):169-191, 2007. [p83]

K. Boudt and J. Cornelissen. RTAQ, 2012. URL http://cran.r-project.org/src/contrib/Archive/ RTAQ/. R package version 0.2. [p81]

K. Boudt, P. Carl, and B. G. Peterson. PortfolioAnalytics: Portfolio Analysis, including Numeric Methods for Optimization of Portfolios, 2010. URL http://r-forge.r-project.org/projects/returnanalytics/. R package version 0.6. [p80]

J. Cornelissen, K. Boudt, and S. Payseur. highfrequency, 2013. URL http://CRAN. R-project.org/ package=highfrequency. R package version 0.2. [p80,81]

D. Easley and M. O'Hara. Price, Trade Size, and Information in Securities Markets. Journal of Financial Economics, 19(1):69-90, 1987. [p80]

D. Easley, N. M. Kiefer, M. O'Hara, and J. B. Paperman. Liquidity, Information, and Infrequently Traded Stocks. Journal of Finance, 51(4):1405-1436, 1996. [p80, 81, 82, 83]

D. Easley, S. Hvidkjaer, and M. O'Hara. Is Information Risk a Determinant of Asset Returns? Journal of Finance, 57(5):2185-2221, 2002. [p80, 83]

D. Easley, R. F. Engle, M. O'Hara, and L. Wu. Time-Varying Arrival Rates of Informed and Uninformed Traders. Journal of Financial Econometrics, 6(2):171-207, 2008. [p83, 84]

D. Easley, M. M. L. de Prado, and M. O'Hara. Flow Toxicity and Liquidity in a High Frequency World. Working paper, 2012. [p84]

J. Enos, D. Kane, A. R. Narayan, A. Schwartz, D. Suo, and L. Zhao. Trade Costs. R News, 8(1):10-13, 2008. [p81]

L. R. Glosten and P. R. Milgrom. Bid Ask and Transaction Price in a Specialist Market with Heterogeneously Informed Traders. Journal of Financial Economics, 14(1):71-100, 1987. [p81]

J. Hasbrouck and D. J. Seppi. Common Factors in Prices, Order Flows and Liquidity. Journal of Financial Economics, 59(1):383-411, 2001. [p81]

T. Hendershott and A. J. Menkveld. Price Pressures. Unpublished version, 209, 1991. [p85]

J. Idier and S. Nardelli. Probability of Informed Trading on the Euro Overnight Market Rate. International Journal of Finance and Economics, 16(2):131-145, 2011. [p80]

D. Kane, A. Liu, and K. Nguyen. Analyzing an Electronic Limit Order Book. The R Journal, 2(64-68):1, 2011. [p 80,81$]$

A. S. Kyle. Continuous Auctions and Insider Trading. Econometrica, 53(6):1315-1335, 1985. [p81]

C. M. C. Lee and M. J. Ready. Inferring Trade Direction from Intraday Data. Journal of Finance, 46(2): 733-746, 1991. [p80, 81]

H. Li, J. Wang, C. Wu, and Y. He. Are Liquidity and Information Risks Priced in the Treasury Bond Market? Journal of Finance, 64(1):467-503, 2009. [p80]

J. A. Ryan. IBrokers, 2011. URL http: //CRAN. R-project. org/package=IBrokers/. R package version 0.9-10. [p81]

K. Venkataraman. Automated Versus Floor Trading: An Analysis of Execution Costs on the Paris and New York Exchanges. Journal of Finance, 56(2):1445-1485, 2001. [p81] 
P. Zagaglia. PIN: Estimates the Parameters of a Trading-tree Model for the Computation of the Probability of Informed Trading, 2013. URL http://CRAN. R-project. org/package=PIN. R package version 0.8. [p80]

Paolo Zagaglia

International Entrepreneurship Academy

Dublin, Ireland

Rimini Centre for Economic Analysis and

Department of Economics and Department of Cultural Goods, Università di Bologna

Bologna, Italy

paolo.zagaglia@gmail.com 